\title{
Broadcasting in Prefix Space: P2P Data Dissemination with Predictable Performance*
}

\author{
Matthias Wählisch ${ }^{\dagger}$ \\ Freie Universität Berlin \\ Institut für Informatik \\ Takustr. 9 \\ D-14195 Berlin, Germany \\ waehlisch@ieee.org
}

\author{
Thomas C. Schmidt \\ HAW Hamburg \\ Dept. Informatik \\ Berliner Tor 7 \\ D-20099 Hamburg, Germany \\ t.schmidt@ieee.org
}

\author{
Georg Wittenburg \\ Freie Universität Berlin \\ Institut für Informatik \\ Takustr. 9 \\ D-14195 Berlin, Germany \\ wittenbu@inf.fu-berlin.de
}

\begin{abstract}
A broadcast mode may augment peer-to-peer overlay networks with an efficient, scalable data replication function, but may also give rise to a virtual link layer in VPNtype solutions. We introduce a generic, simple broadcasting mechanism that operates in the prefix space of distributed hash tables without signaling. This paper concentrates on the performance analysis of the prefix flooding scheme. Starting from simple models of recursive $k$-ary trees, we analytically derive distributions of hop counts and the replication load. Further on, extensive simulation results are presented based on an implementation within the OverSim framework. Comparisons are drawn to Scribe, taken as a general reference model for group communication according to the shared, rendezvous-point-centered distribution paradigm. The prefix flooding scheme thereby confirmed its widely predictable performance and consistently outperformed Scribe in all metrics. Reverse path selection in overlays is identified as a major cause of performance degradation.
\end{abstract}

Keywords: Prefix flooding, DHT, random recursive $k$ ary trees, overlay network simulation, Pastry, Scribe

\section{Introduction}

A broadcast service is commonly supported on the network and data link layer. Analog to the IP layer, application overlays may require the use of an unselective group communication. Distributed Hash Tables (DHT) like Chord [12]

\footnotetext{
*This work is supported in part by the German Bundesministerium für Bildung und Forschung within the project Moviecast (http://moviecast.realmv6.org).

† The author is also with HAW Hamburg, Dept. Informatik, and with link-lab, Berlin.
}

and Pastry [11] do not consider broadcast, i.e., a mechanism to communicate to all parties of one DHT instance without their active participation.

The broadcast mode admits two unique features. The a priori awareness of the data flooding task may significantly enhance efficiency, e.g., by taking advantage of network or (shared) media specifics. Further on, it enables a message exchange among mutually unknown parties without a requirement of specific service awareness or any form of signaling. Broadcast is thus the fundamental mechanism for unselective data synchronization and for the autonomous coordination of distributed systems.

On the application layer, there are likewise versatile use cases for broadcast communication. Applications range from broadband data dissemination in video conferencing or data replication, over service and peer discovery up to the implementation of a virtual link layer in VPN-type solutions.

Broadcast is a special case of multicast. This distribution mechanism guarantees to reach not only a subset, but all nodes of a dedicated domain without explicit registration. The set of all nodes is also called the broadcast domain. It is worth noting that a broadcast domain can be arranged on different layers with varying inherent capabilities. Connecting nodes, e.g., with an Ethernet hub to a shared segment facilitates packet distribution based on the physical network structure. It is limited by the supporting medium, i.e., the range of signal propagation. The equivalent holds for the wireless domain, where the medium is always shared, but of restrictive propagation ranges. Participating nodes do not need a specific network logic in sending and receiving broadcast data on the physical layer. Broadcast support, however, on a dedicated layer should be independent of the underlying tier, which may accelerate it. In the example of IP, broadcast addresses will be directly mapped to the Ethernet broadcast address, such that all Ethernet hosts 
of one segment receive the data independent of their subnet assignment, but in contrast to network access, packets can be forwarded on the network layer beyond physical bounds.

In general, broadcast in logical networks can be enabled by passing data incrementally to direct overlay neighbors. If the graph of nodes is connected and contains the source, all nodes will be reached. DHT structures allow to derive such a connected neighborhood graph. Any node can send packets to an address adjacent to its own key space. In contrast to IP, every possible address is associated with one overlay peer. Such a simple ring broadcast scheme sends the packet to exactly one neighbor, reaching all $n$ DHT peers after $n$ hops. As an alternative approach to the case of unknown neighborhoods, a dedicated, well-known replicator can be placed in the network like the Broadcast and Unknown Server in ATM. Such a rendezvous point-based approach requires extra signaling to register receivers. The parallelism of distribution is bounded by the replicator, which sustains the overall duplication load and may be a single point of failure.

In the following, we will present a general broadcast algorithm along with optimizations for Pastry, that uses the DHT structure more efficiently and replicates data stepwise to all neighbors in prefix space. This scheme works without peer involvement, especially without signaling. We model and analyze the approach theoretically and in simulation, drawing comparison to a generic rendezvous point approach derived from Scribe [3].

This paper at first gives an introduction of the prefix flooding algorithm in the next section and continues as follows. Section 3 presents an overview of the performance measures applied in our analysis, while analytical models are utilized in section 4 to derive distributions for the core properties of replication load and hop count. Results of our simulation studies are outlined in the subsequent section 5 . Related work is reviewed in section 6 , followed by a final discussion and conclusion in section 7 .

\section{Broadcast by Prefix Flooding}

For an efficient application layer broadcast we need to define a strategy for data replication on the overlay. In a DHT, the peer identifiers are composed using an alphabet of $k$ digits and have a predefined length. All nodes of a structured overlay can be naturally arranged in a prefix tree, branching recursively at longest common prefix of $k$ neighboring vertices. The leaves are labeled with the overlay identifiers of the DHT members and the inner vertices represent the shared prefix (cf. figure 1).

This tree can be interpreted as a distribution tree, defining the broadcast domain of a specific DHT instance. If a broadcast packet is sent starting from the root of the tree towards the leaves, the packet will be replicated where pre-

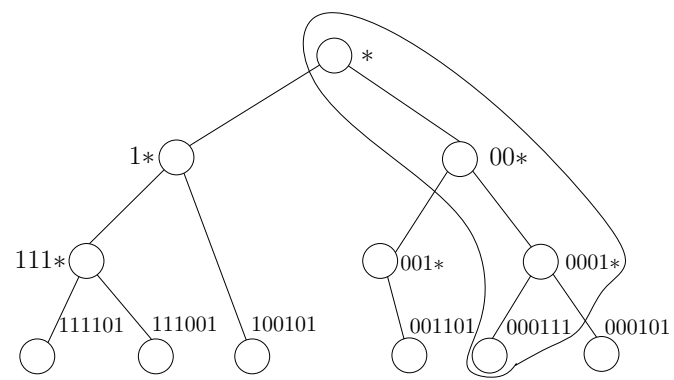

Figure 1. DHT Node within a Prefix Tree Associated Vertices are Highlighted.

fixes branch. Actually, the broadcast domain (prefix tree) decomposes in many smaller broadcast sub-domains (subtrees), in which the propagations continue in parallel. Following the nature of broadcast, a packet will be forwarded locally, after it has arrived at a root of a subtree.

This approach allows to reach all peers of a DHT, because the data is flooded to the leaves, which represent the overlay nodes. A peer receiving a broadcast is required to determine the current branching position on the distribution tree to decide on further packet replication. This context awareness can be achieved by sending broadcast packets carrying the prefix currently addressed, which we call destination prefix. This destination prefix will grow in length with every forwarding hop while descending the tree.

We denote the length of a prefix $\mathcal{A}$ by $|\mathcal{A}|$. Given two prefixes $\mathcal{A}$ and $\mathcal{B}$, the longest common prefix will be written $\mathcal{L}=L C P(\mathcal{A}, \mathcal{B})$. The relation of $\mathcal{L}$ being a prefix of $\mathcal{A}$ is written as $\mathcal{L} \subseteq \mathcal{A}$. Consequently $\mathcal{L} \subseteq \mathcal{A}$ and $\mathcal{A} \subseteq \mathcal{L}$ if and only if $\mathcal{L}=\mathcal{A}$.

A proper specification for data distribution, i.e., a routing procedure on prefix trees, requires further definitions. The two sub-problems that need to be solved are $a$ routing to a prefix and the association of nodes with prefixes:

Definition 1 A prefix $\mathcal{L}$ is associated with an overlay node of ID $\mathcal{N}$, if and only if $\mathcal{L} \subseteq \mathcal{N}$.

As shown in figure 1, all inner vertices on the shortest path from the root to a node are associated with that node.

Concordantly, a prefix routing can be defined as forwarding a packet to the node the destination prefix is associated with. In general, there may be several nodes owning an associated prefix, since prefix-to-node mapping is only assured to be unique for prefixes of full key length. For flooding a prefix tree, a forwarding peer needs to route packets to all 'live' neighboring prefixes (cf. figure 1). Consequently, a peer must store corresponding nodes for each prefix adjacent to its associated vertices in a prefix neighbor set. It is important that these tables are complete. A complete neighbor set meets the following condition: Whenever an over- 
lay node exists for a given prefix, then the neighbor set will provide an entry for this prefix. In particular it follows that each overlay node is a destination in at least one set, since node keys are uniquely assigned. It is worth noting that a prefix needs not to be included in any neighbor set, if there is no peer sharing it. The requirement of complete neighbor tables will usually be fulfilled by the key-based routing service, i.e., underlying DHT routing maintenance.

A source initiates a broadcast by starting with the empty destination prefix. This corresponds to delivering the data to all prefix neighbors $\mathcal{N}_{i}$. At each neighbor a packet will be further replicated. The destination prefix is replaced with the new target address. In detail, the algorithm works as follows:

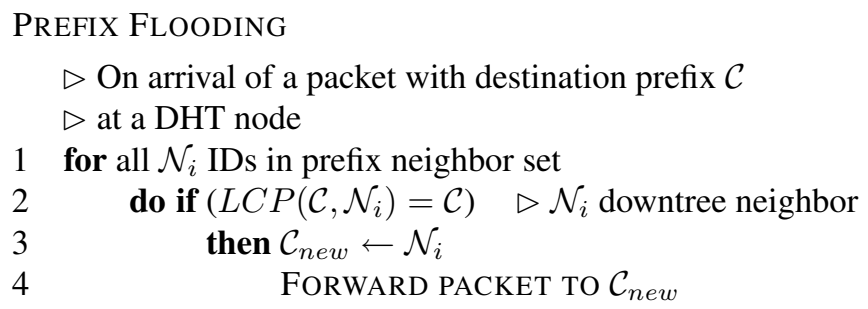

If an inner vertex of the prefix tree fails, e.g., due to churn, the corresponding sub-tree is empty or includes further peers. The replacement of the next hop for a given prefix $\mathcal{C}_{\text {new }}$ will be achieved by the underlying DHT. In general, in the case of overlay network failures the reliability of prefix flooding relies directly on the deployed DHT maintenance.

If all peers have a complete set of prefix neighbors, the scheme guarantees that all overlay nodes will be accessed, no peer receives a broadcast packet more than once and the algorithm terminates.

Theorem 1 (Coverage) If the prefix neighbor sets are complete at all nodes, then the PREFIX FLOODING assures packet distribution to all overlay nodes.

Theorem 2 (Uniqueness) Each overlay node will receive a broadcast packet at most once using the PREFIX FLOODING.

Complete proofs for both theorems are elaborated in [14]. Theorem 1 can be proven by induction over the number of overlay nodes, while theorem 2 follows from the observation that each routing prefix uniquely identifies the root of a subtree in prefix space.

From theorem 2 it can be concluded that the PREFIX FLOODING does not induce loops, proving the assumption that the algorithm terminates.

\subsection{Implementation for Pastry}

The idea of prefix routing is implemented in Pastry. The Pastry routing table of a peer reflects directly the elements of a prefix tree. Thus each peer carries a subset of the prefix tree in its routing table. Merging the routing tables of all peers, would form the global distribution tree. In flooding their routing tables, Pastry peers flood the prefix tree, which corresponds to the overlay broadcast described by the PREFIX FLOODING. In detail, the idea is as follows: A source sends its data to all routing table entries. Each destination prefix corresponds to the root of a broadcast sub-domain. The receiving peers determine their position in the tree, i.e., the height $D$ in the prefix tree, at which they receive the data, and forward the packets downwards. This is equal to sending data to all routing table entries starting at row $D+1$. Note that the tree position can easily be derived by denoting the row number, which reduces the packet size in contrast to encoding the entire key. For Pastry the PREFIX FLOODING reads in pseudo code:

$$
\begin{aligned}
& \text { PASTRY PREFIX FLOOdING } \\
& \triangleright \text { On arrival of a packet with destination prefix length } \\
& \triangleright D \text { at Pastry node of ID } \mathcal{K} \text { with routing table } A \\
& \triangleright \text { containing } l \text { rows and } k \text { columns } \\
& 1 \text { for all } i \leftarrow D+1 \text { to } l \\
& 2 \quad \text { do for all } j \leftarrow 1 \text { to } k \\
& 3 \text { do if } a_{i, j} \neq \text { Unspecified } \wedge a_{i, j} \neq \mathcal{K} \\
& 4 \quad \text { then } D_{\text {new }} \leftarrow i \\
& 5 \quad \text { FORWARD PACKET TO } a_{i, j}
\end{aligned}
$$

If the routing table is filled correctly, all theorems for the PREFIX FLOODING are also valid for Pastry, since the Pastry routing table corresponds to the set of prefix neighbors $\left\{\mathcal{N}_{i}\right\}$. However, Pastry reactive maintenance does not guarantee that each overlay node will provide complete routing states [11], which conflicts with the PREFIX FLOODING. Therefore we augmented Pastry with a proactive routing maintenance mechanism, which performs initial key lookups to fill the routing table similar to the "fix_fingers" routine in Chord.

\section{Performance Measures}

The prefix flooding approach to broadcasting introduces prefix trees as a control plane to packet forwarding. This simple mechanism operates without additional signaling, which is an apparent advantage. The quality of the routing as inherited from a hash-generated prefix tree needs closer inspection. Ideally, packet distribution should be fast and minimize traffic and replication load in the network. To obtain an overall insight into the routing quality, we evaluate the prefix flooding scheme in theory and in a discrete event 
simulation according to the following metrics and compare our results to Scribe [3]. Scribe serves as a generic reference model for schemes using dedicated replicators, and is based on the same DHT, Pastry. It is worth noting that the performance metrics do not measure the multicast specific properties of Scribe. Thus, choosing Scribe for comparison is reasonable.

Packet replication load quantifies the number of packets a single peer has to forward. This metric reflects the number of direct neighbors per node in the distribution tree. The overall characteristic for the prefix routing is then given by the distribution of the replication load obtained from all forwarding nodes.

Hop count counts the number of overlay routing traversals that a packet needs on its way from the source to the destination. Note that the hop count affects the travel time, because every additional hop results directly in an additional transmission time. In this sense the travel time is correlated with the hop count.

Travel time describes the time a data packet travels from the source until it reaches a receiver measured in seconds. This absolute value depends on the one hand on the number on hops between the nodes and on the other hand of the transmission time inherited from the hop by hop link delays and the packet size of the transmitted data.

Relative delay penalty measures the ratio of the travel time for data packets delivered via Scribe and the travel time resulting from the prefix flooding scheme. This relative factor gives an indication of the parallelism of packet forwarding.

\section{Analytical Models}

To understand the performance of the prefix flooding scheme, we first present analytical considerations. Based on the shape of the prefix tree, we gain insight in the structural behavior of protocols for traversing prefix distribution trees. As this analysis is only based on the tree itself, fringe effects known from simulations are isolated.

\subsection{Replication Load}

In the following, we want to derive the distribution of the replication load in a prefix tree. For the general case of prefix flooding in a structured overlay of $N$ nodes using a prefix alphabet of $k$ digits, the following upper bound of the replication load can be derived immediately.

Theorem 3 Any overlay node in a prefix flooding domain of $N$ receivers and an alphabet with $k \geq 2$ digits will replicate a data packet at most $\log _{2}(N)(k-1)$ times.

For the distribution function of the replication load in a fully populated prefix tree, we need to determine replication

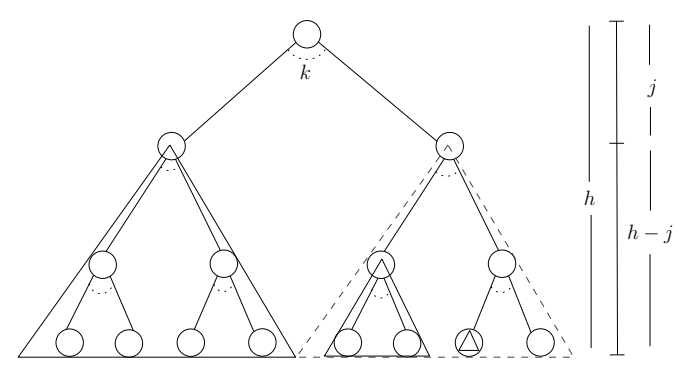

Figure 2. Self-Similarity of Prefix Subtrees due to the Recursive Nature of $k$-ary Trees

values along with their frequencies. Recalling the picture of a full prefix tree for an alphabet with $k$ digits, every node except the leaves has $k$ children. The number of packet replications for an overlay peer is equal to the overall number of forwarding neighbors, which depends on the tree position, where a peer receives the packet. Per level the replication load is $k-1$. Consequently, in a fully populated $k$-ary prefix tree of height $h$, replication occurs only at multiples of $k-1$, the number of neighbors in prefix space. For $j \geq 0$ we denote these discrete values by $v_{h, k}(j)=(h-j)(k-1)$.

To derive the replication frequency, we quantify the occurrence of the replication load $v_{h, k}(j)$. Since we know the load of a peer forwarding packets at height $j$, the frequency can be calculated by counting the number of peers that fulfill the replication condition. The latter corresponds to the number of (sub-)trees with height $h-j$, because every peer serves as forwarder for one tree. Starting at the source in a full prefix tree, the structure decomposes in $k-1$ subtrees with height $h-1, k(k-1)$ subtrees of height $h-2$, etc. (cf. figure 2). At every level of the full prefix tree, there is an exponential growth in the number of inner vertices representing the root of new subtrees. Thus, the frequency of $(h-j)$-size subtrees must increase exponentially with their decreasing height. In detail there are $k^{j-1} \cdot(k-1)$ subtrees of height $h-j$, which account for a replication load of $(h-j) \cdot(k-1)$.

Theorem 4 Given a fully populated $k$-ary prefix tree of height $h$. Then the frequency $f_{h, k}\left(v_{h, k}(j)\right)$ for a replication load $v_{h, k}(j)=(h-j)(k-1)$ is given by

$$
f_{h, k}\left(v_{h, k}(j)\right)= \begin{cases}1 & \text { for } j=0 \\ k^{j-1} \cdot(k-1) & \text { for } 0<j \leq h .\end{cases}
$$

Proof by induction. We assume a full $k$-ary prefix tree of height $h$. The case $j=0$ corresponds to the (single) source that replicates data to $h(k-1)$ neighbors as derived above.

The induction is done with respect to $h-j$, the height of a subtree (cf. fig. 2).

Base case: Is $h-j=1$, we have to show that the replication load $v_{h, k}(h-1)$ appears $(k-1)$-times. In a tree 
of height 1 , the source sends the data to all further leaves directly, which equals $k-1$.

Induction step: Assume the statement holds for $h-j$. We have to show that the statement holds for $h-j+1$, i.e., $f_{h, k}\left(v_{h, k}(h-j+1)\right)=k^{h-j}(k-1)$.

Consider a full prefix tree of height $h-j+1$. It consists of $k$ subtrees of height $h-j$. The replication load of a node in a tree of $(h-j+1)$ equals the sum of all neighbors in these $\mathrm{k}$ subtrees. Using the induction hypothesis the overall replication load reads

$k \cdot f_{h, k}\left(v_{h, k}(h-j)\right)=k k^{h-j-1}(k-1)=k^{h-j}(k-1)$.

The overall number of packet replications is easily identified as the number of leave nodes, since there are no packet duplications and each peer receives the broadcast. The number of leaves of a full $k$-ary tree of height $h$ equals $k^{h}$, such that we arrive at the following

Corollary 1 The probability distribution $P_{h, k}$ for packet replication multiplicities reads

$$
P_{h, k}\left(v_{h, k}(j)\right)= \begin{cases}k^{-h} & \text { for } j=0 \\ k^{j-h-1} \cdot(k-1) & \text { for } 1 \leq j \leq h \\ 0 & \text { otherwise. }\end{cases}
$$

Corollary 2 The average replication load for a node in a full prefix tree $T_{h, k}$ is given by $1+\mathcal{O}\left(k^{-h}\right)$, its standard deviation by $\sqrt{k}+\mathcal{O}\left(k^{-h}\right)$.

Observing the weak dependence of the replication load distribution on $h$ and $k$, i.e., the tree shaping parameters, it can be assumed that the model is sufficiently general to grant insights into the qualitative replication behavior of a sparsely populated $k$-ary trees. We will see in section 5 that the simulations support this assumption.

\subsection{Hop Count}

As for the replication load, we firstly derive general measures of the number of hops a packet travels from the source to any destination in the prefix flooding scheme.

Theorem 5 Any overlay node in a structured broadcast domain of $N$ receivers and an alphabet with $k \geq 2$ digits will receive a packet from prefix flooding after at most $\log _{2}(N)$ hops. In the presence of Pastry overlay routing, the number of hops attained on average equals $\log _{2^{b}}(N)$ with $k=2^{b}$.

We now want to return to considering a fully populated prefix tree and derive the hop distribution thereof. The main idea is similar to the replication load: A forwarding peer sends the broadcast to $k-1$ prefix neighbors, all of them rooting an equally structured subtree of height $h-1$. We are counting the number of paths with a length reduced by one herein. Additionally we count the frequency of paths for the calculated hop count in the virtual subtree containing the forwarder. This recursion results in

Theorem 6 Given a fully populated $k$-ary prefix tree of height $h$, the frequency $f_{h, k}(j)$ of a hop count $j$ occurring in prefix flooding is given by

$$
f_{h, k}(j)=\left(\begin{array}{c}
h \\
j
\end{array}\right)(k-1)^{j}
$$

Proof. A flooding packet arriving at node $n$ after $j$ hops will admit a current destination prefix of length $j$. Being located in a subtree of height $h-j, n$ will forward the packet to its downtree neighbors, thereby partitioning its subtree into $k-1$ further subtrees of height $h-j-1$ (cf. figure 2). Due to the recursive nature of the $k$-ary prefix tree, the frequency distribution satisfies the recurrence relation

$$
f_{h, k}(j)=f_{h-1, k}(j)+(k-1) \cdot f_{h-1, k}(j-1)
$$

with initial conditions $f_{1, k}(0)=1, f_{1, k}(1)=k-1$. Inserting $f_{h, k}$ yields the claim.

This result can be interpreted in two different ways. Among all legitimate paths in downtree routing, i.e., of length $h$, those of length $j$ are selected and branch $k-1$ times at each of the $j$ intermediate prefix nodes. Alternatively, flooding corresponds to a node discovery process, where a node discovers its $v_{h, k}(j)=(h-j)(k-1)$ neighbors which in turn discover their neighbors in the following step. Subsequent neighbor discovery requires connect to the $j$-th part as only $(h-j)(k-1) / j$ nodes have further neighbors.

Following a similar argument as in corollary 1 , it is clear that normalization for hop count frequencies is given by $k^{h}$, the number of leaf nodes in the full prefix tree.

Corollary 3 The probability distribution $H_{h, k}(j)$ of the hop count for flooding a full prefix tree $T_{h, k}$ evaluates to

$$
H_{h, k}(j)=k^{-h} \cdot\left(\begin{array}{l}
h \\
j
\end{array}\right)(k-1)^{j} .
$$

Corollary 4 The average hop count at which a packet is received from flooding in a full prefix tree $T_{h, k}$ is given by $<H_{h, k}>=(k-1) / k \cdot h$, the standard deviation of the hop count distribution (5) equals $\sigma_{H_{h, k}}=\sqrt{(k-1) \cdot h} / k$. 
This average is almost independent of the prefix alphabet $k$ and can be in some sense interpreted as the counterpart of the average replication load as seen in corollary 2. As the average number of per hop replications is close to one, packets travel down the entire tree and reach most of their receivers after nearly $h$ hops. The width of the hop count distribution, its standard deviation, admits a weak dependence on $k$, slowly decaying from its maximum at $k=2$ as $k^{-1 / 2}$.

In contrast to the replication load distribution, which showed only a weak dependence on the tree shaping parameters, the hop count results strongly depend on $h$ for the fully populated k-ary tree. The height $h$ is directly related to the number of nodes $k^{h}$ in this tree, which does not hold for realistic scenarios. Thus a direct transfer to sparsely populated random trees is questionable.

To derive a distribution for general distribution trees, evaluations are required on the class of all random k-ary trees. Unfortunately, this turns out to be difficult. Proceeding in a significantly simpler, but reasonable approach, we restrict the analysis to the class of random recursive $k$ ary trees with a homogeneous probability $p$ for independent edges. In this model, each vertex branches to each of its $k-1$ possible outdegrees independently with probability $p$, thereby preserving the recursive nature of the fully populated $k$-ary tree. Instead of equation 4 , the hop frequency of routing on this random recursive tree will be governed by the modified rate equation

$$
\begin{aligned}
f_{h, k}(j)= & f_{h-1, k}(j)+p \cdot(k-1) \cdot f_{h-1, k}(j-1) \\
& \text { with } f_{1, k}(0)=1, f_{1, k}(1)=p(k-1) .
\end{aligned}
$$

This can be solved analogously to 4 and yields

Corollary 5 The probability distribution $H_{h, k}^{(p)}(j)$ of the hop count for flooding a random recursive k-ary prefix tree $T_{h, k}^{(p)}$ with homogeneous, independent edge probability $p$ evaluates to

$$
H_{h, k}^{(p)}(j)=(1+p(k-1))^{-h} \cdot\left(\begin{array}{c}
h \\
j
\end{array}\right) \cdot(p(k-1))^{j},
$$

which attains the average value $\left\langle H_{h, k}^{(p)}>=\frac{p(k-1)}{1+p(k-1)} \cdot h\right.$, and the standard deviation $\sigma_{H_{h, k}^{(p)}}=\frac{\sqrt{p(k-1) \cdot h}}{1+p(k-1)}$.

The introduced edge probability $p$ is not a 'free' parameter, but a function of the number of leaf nodes $N=$ $(1+p(k-1))^{h}$ in the tree. Solving this relation for $p=\frac{\sqrt[h]{N}-1}{k-1}$, and inserting typical Pastry parameters for $k=16, h=128$ and node numbers of our simulations, will lead to the relatively small edge probabilities, mean hop counts and standard deviations displayed in table 1 .

\begin{tabular}{|c||c|c|c|c|}
\hline \multicolumn{1}{|c||}{} & \multicolumn{4}{c|}{$k=16, h=128$} \\
\hline$N$ & 10 & 100 & 1.000 & 10.000 \\
\hline \hline$p$ & 0.00122 & 0.00244 & 0.00370 & 0.00497 \\
\hline$<H_{h, k}^{(p)}>$ & 2.30 & 4.52 & 6.73 & 8.88 \\
\hline$\sigma_{H_{h, k}^{(p)}}^{(p)}$ & 1.50 & 2.09 & 2.53 & 2.87 \\
\hline
\end{tabular}

Table 1. Selected Link Probabilities, Mean Hop Counts and Standard Deviations.

These analytical results will not only support a qualitative insight into the mechanisms of prefix-based packet distribution, but will also show significant agreement with the simulation results presented in the subsequent section.

\section{Simulation Results}

In this section, we will analyze the performance of the prefix flooding based on a stochastic discrete event simulation and compare to the behavior of the rendezvous pointbased approach Scribe. Both, the prefix flooding and Scribe, are implemented on top of a proactive version of the DHT substrate Pastry.

In detail, our simulations are performed on the network simulator platform OMNeT++ 3.3 [13], supplemented by a preliminary version of the overlay simulation package OverSim [2] including Scribe and extended by the prefix flooding implementation. Pastry has been configured as in its original version [11]. Especially, we use a key length of 128 and an alphabet size of 16 , if not mentioned otherwise. To investigate the scaling behavior of the protocols, the simulations are conducted for a number of peers varying by three orders of magnitude. None of the relative metrics described in section 3 depend on the underlay. Thus the Simple model [1] has been applied as the underlying network with a homogeneous link delay of $1 \mathrm{~ms}$ to analyze the network properties inside the overlay.

The analysis is not focusing on reliability aspects, which allows us to neglect churn. In particular, any effects of volatile nodes would be completely maintained by Pastry for the prefix flooding and partially for Scribe. Rendezvous point (RP) based schemes have to reorganize the distribution tree due to failing RPs, resulting in DHTs by new key associations, which nevertheless is not addressed here.

Summarizing the simulation scenario, we calculate the flooding performance on an arbitrary $(k=16)$-ary prefix tree with a fixed maximal height and a varying number of leaves interconnected by links of identical weight. The broadcast will be initiated by a randomly selected leaf. 


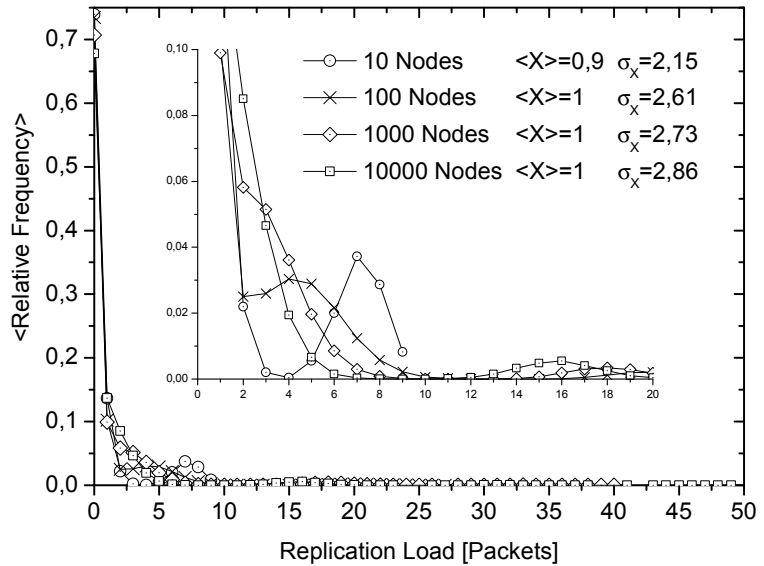

(a) Prefix Flooding, $k=16$

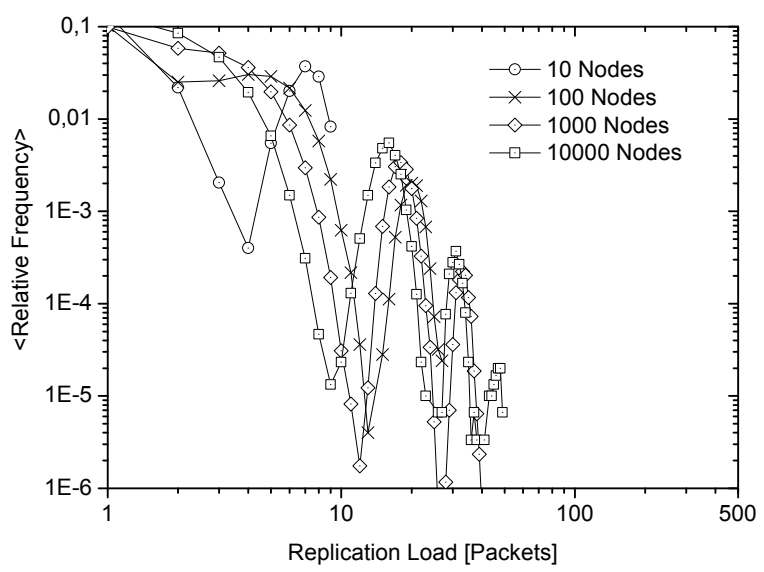

(c) Detail: Tail for Prefix Flooding, $k=16$

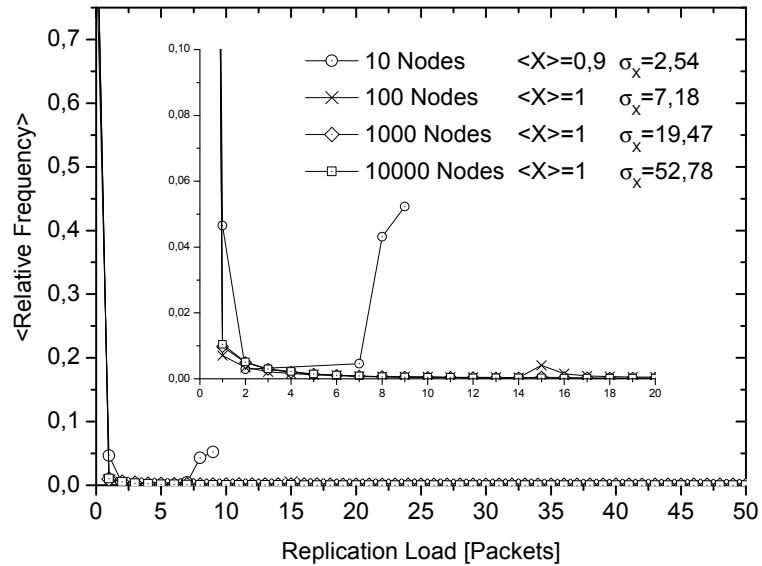

(b) Scribe, $k=16$

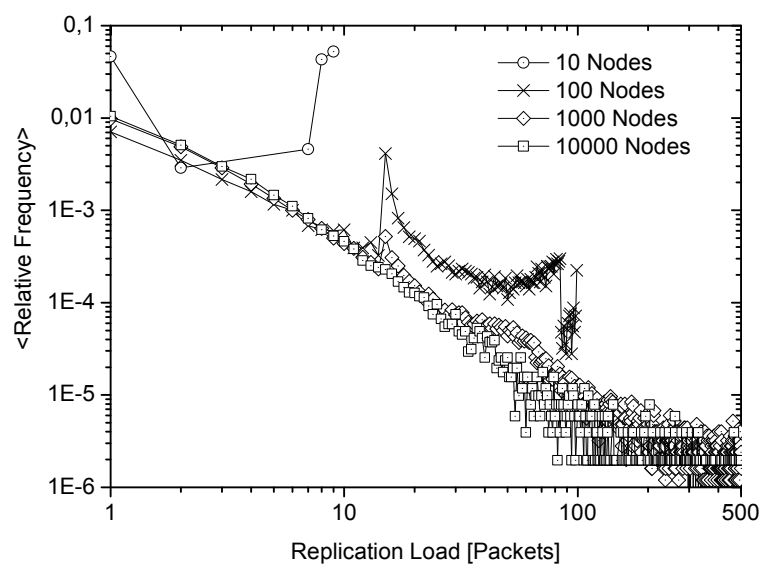

(d) Detail: Tail for Scribe, $k=16$

Figure 3. Distribution of Packet Replication Comparing Prefix Flooding with Scribe

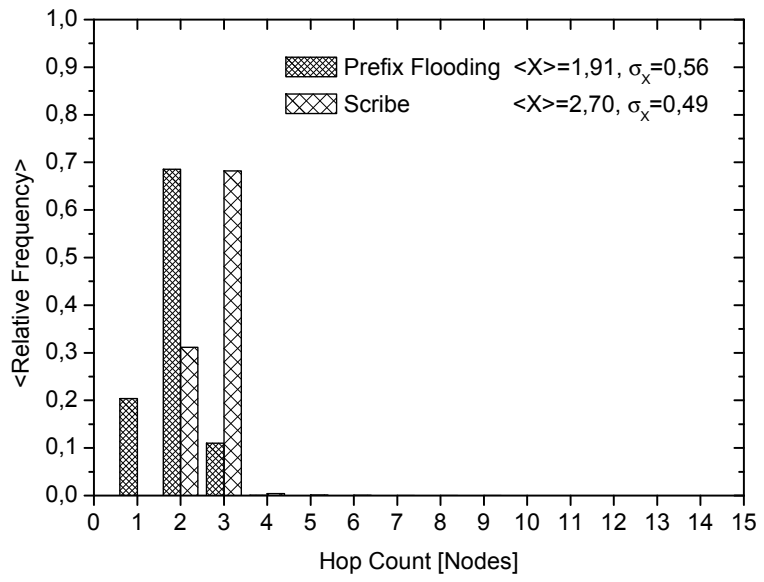

(a) $N=100$

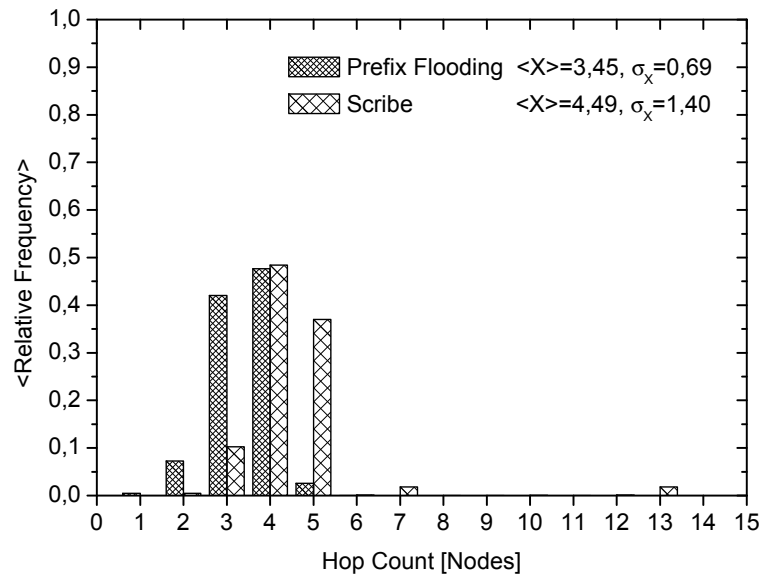

(b) $N=10000$

Figure 4. Hop Count Distribution for an Overlay of Size $N$ 


\subsection{Replication Load}

The distributions of the peer replication load for prefix flooding and Scribe are displayed in figure 3. Both schemes show an exponential decay around their common average value of 1 . However, the shapes of the distributions for the two approaches vary significantly, which becomes apparent at first from standard deviation values. While the widths of the distributions for prefix flooding are small und almost independent of network sizes, the corresponding values for Scribe grow large, about linearly in the number of nodes.

Both broadcasting schemes produce a large number of replications of values 0 and 1 , but frequencies drastically drop for higher multiplicities. Prefix flooding distribution attains a much smoother decay, leaving significant probability to replication values of $2-10$. Smoothness is even more pronounced for smaller alphabets, which for space restrictions are omitted here. In contrast, Scribe decreases faster from its average, decaying rapidly to probabilities below $1 / 100$ for replications larger than 2 , fairly independent of the alphabet $k$.

An exception from this overall shape can be observed for the distribution of 10 peers in Scribe. Here, the frequencies of replication values around 9 are strongly enhanced. This border effect for very small networks can be understood from analyzing distribution tails. As visualized in the log-log plot 3(d), the distribution of Scribe is heavytailed according to a power law decay, representing remarkably high probabilities for very large replication values up to 7800 . Corresponding probabilities are accumulated for small sized overlays.

In contrast, the prefix flooding distribution admits a strict exponential decay, with tail weights vanishing above 50 . Replication values in prefix flooding are superimposed by oscillating frequencies as visible in figure 3(c). The resulting probability "bumps" are noticeable on different scales for all overlays and can be explained by our theoretical analysis, which reveals an exponential decay within the range of multiples of $(k-1)$. Compared to the prerequisites of corollary 1 , the simulated overlays do not operate on full $k$-ary prefix trees. Hence replication values do not only occur as multiples of the branching factor, but level out with neighboring values. Nevertheless, regarding the peaks of the bumps, the population and replication pattern of the $\mathrm{k}$ ary trees remain clearly visible.

In both approaches, most of the peers receive the broadcast without a need to forward it further. Scribe thereby stresses a small number of peers to serve a much higher replication load. Instead, the prefix flooding reduces the maximal replication load by distributing the load evenly over the neighbors.

\subsection{Hop Count}

The mean hop count distribution for different overlay sizes is shown in figure 4. In general, both schemes show the logarithmically growing hop path length dependent on the number of peers. With an increasing quantity of leaves, the height of prefix trees will increase logarithmically, as well, resulting in longer paths from the source and intermediate forwarders to the receivers. The mean hop count $\langle X\rangle$ for Scribe highlights approximately one additional node in contrast to the prefix flooding.

For a sufficiently large $N>10$, the average of the distribution for the prefix flooding attains directly the calculated mean hop count in theorem 5, at which all other hop count values are centered. The hop count distribution in Scribe shows a heavy-tailed behavior, which increases with the overlay size as indicated by the approximate linear growth of the standard deviation. In contrast, the prefix flooding almost attains a constant variation. Consequently, in prefix flooding the path lengths are tightly concentrated around the logarithmically bounded average, while Scribe builds up longer branches with higher weights.

\subsection{Relative Delay Penalty}

Figure 5 shows the relative delay penalty (RDP) as function of the network size for Scribe over prefix flooding. Scribe packets travel about a factor of 1.4 slower than data of prefix flooding in larger networks. The enhanced delay penalty in small networks of about 10 peers reflects the observations of figure 3(b) that almost all receivers are addressed directly by the rendezvous point, which replicates the full number of overlay nodes. The more keys are allocated, the more branching points are located close to the RP resulting in longer paths and less efficient parallelism in Scribe, which is in contrast to the prefix flooding.

\section{Related Work}

The principal approach for implementing broadcast on a pure DHT derives from recursive partitioning of the key space with data distribution following partition ranges. The prefix flooding operates in this sense, defining numerical interval boundaries from prefix transitions. The first idea of a broadcast based on nested intervals was proposed in [5]. The broadcast is sent to intervals of exponentially increasing scale as derived from the Chord routing table.

A generalization of [5] is proposed in [6]. In addition to a design independent of Chord, the authors enhance their algorithm by reliability routines, which guarantee a broadcast distribution independent of the routing table states. This is performed by delegating data delivery for missing entries to subsequent forwarders. 


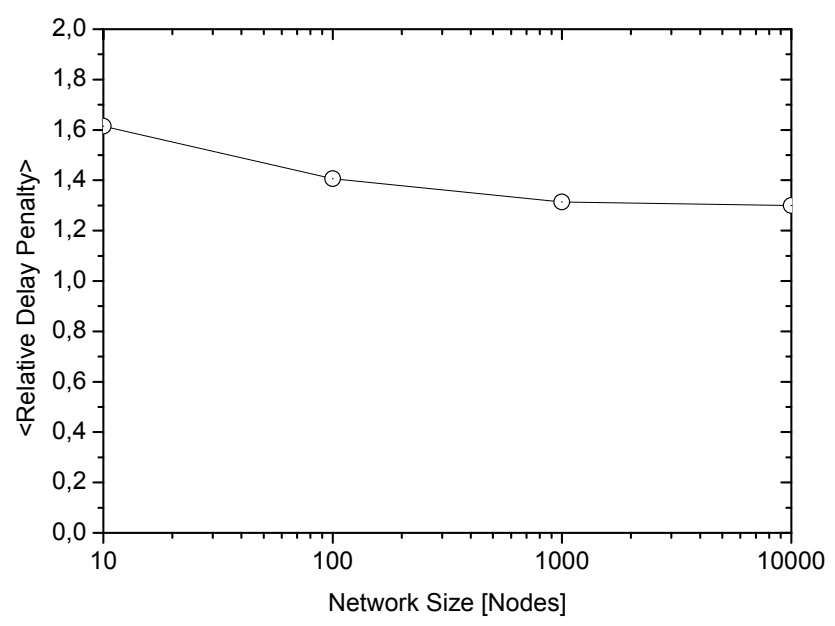

Figure 5. RDP for Scribe over Prefix Flooding

The authors in [8] introduce a scheme, which splits the key space in $d$ partitions of equal size and selects the first node in clockwise direction as the responsible forwarder. Otherwise similar to [5], this approach refrains from using uneven, logarithmic partitioning.

An approach, which cannot ensure a broadcast distribution without data redundancy, is presented in [9]. The authors combine a slightly enhanced version of [5] with an epidemic distribution. All broadcast forwarders send the data periodically to a randomly chosen neighbor, whereby the protocol may duplicate broadcast to the same neighbor. All of the approaches mentioned above lack formal verification, as well as analytical considerations regarding data distribution in $k$-ary prefix trees. Most of the algorithms are implemented on top of Chord, none of them on Pastry, which natively offers a proximity-aware prefix routing.

A generalized construction scheme to partitioning the key is space is presented in [7]. The authors observe that any contractive self-mapping function $P$ of the key space with a single fixed point $\alpha$, i.e., $P(\alpha)=\alpha$, gives rise to a parent relationship. Based on the parent relation $P(\alpha)$, a reverse path can be set up for any node $\alpha$, leading to a broadcast distribution tree with the root $\alpha$. Different parent functions thus give rise to different trees at variable roots, which may be used for load-sharing or redundancy purposes.

DHT specific flooding has been introduced in the early work [10] for CAN (Content Addressable Network). In contrast to Chord or Pastry, CAN maps node IDs to regions representing coordinates in a partitioned $d$-dimensional space. CAN broadcasts the data to all geographical neighbors, thereby accounting for predecessors and foreseeable redundancies. However, the partitioning of the $d$ dimensional space may be uneven and result in data duplication at sub-regions. Performance properties of multicast on CAN are derived analytically in [16]. An extensive simulation study of flooding and tree based overlay multicast over CAN and Pastry with respect to the underlay is presented in [4]. The authors show that CAN flooding is outperformed by Pastry flooding, which relies on a more efficient tree structure adaptive to the underlay.

Our implementation of the generalized prefix flooding is similar to the Pastry flooding of Castro et al. [4]. The main difference lies in the reactive routing maintenance, which may result in data redundancy at the fallback forwarder [4]. The focus of their analysis of broadcast distribution lies in the context of overlay multicast. Results are only based on simulations. The measured metrics reflect performance issues focusing on efforts imposed on the underlying network. In this sense, our work can be understood as complementary: We presented a general prefix flooding and investigate its inherent, structural properties using an analytical model and simulations.

\section{Discussion and Conclusions}

In this work, we have presented and analyzed broadcasting within distributed hash tables. A general prefix flooding approach, distributing data along prefix branches directly to receivers, is compared to a rendezvous point-based scheme which utilizes a shared tree rooted at a predefined anchor peer. Several phenomena of general interest could be observed.

Divergent Path Length Distributions: Our simulation results confirm the mean hop difference of one between the prefix flooding and the rendezvous point-based approach Scribe. This additional, triangular hop in the overlay becomes noteworthy when stretched in the underlay and then may put stress on several links. The major advantage of the prefix flooding, though, is its quite stable concentration of path length distribution around the average, attaining low variations independent of the overlay size. In general, P2P networks consist of volatile nodes. If we assume an overlay with regular churn, i.e., session times in the range of minutes or larger, and a persistent number of peers on average, the DHT moderately reorganizes key associations. Such structural modifications lead to changing paths within the overlay and in the worst case, a single arrival or departure of a node may cause a data path to change drastically. In the prefix flooding, the path length only changes moderately for new and existing peers due to its narrow distribution. In contrast, the heavy-tailed overlay hop count distribution of Scribe produces a largely inhomogeneous travel time, which complicates synchronous applications.

Varying Replication Load: A high variation can also be identified for the packet replication in Scribe. Similar to the prefix flooding, it is rather likely that peers forward with low replication load. Nevertheless, in a long tail distribution nodes are required to replicate many more packets with 
values up to 7.800 in large sized overlays of 10.000 peers. The distribution of packet replication is thus strongly unbalanced, requiring very low and very high values to be served within the same scenario. Such behavior does not only degrade the performance, but may threaten stability and even cause conflicts with intrusion detection systems.

In contrast to Scribe, the prefix flooding guarantees a replication load closely balanced around its average of about 1 . It can be tuned directly by the branching factor $k$. As we know from the theoretical analysis of section 4, packet replications occur as multiples of $k-1$ in full prefix space. Decreasing $k$ adjusts the maximum number of replications to smaller values.

An Overloaded Single Peer: The peers with extraordinarily high packet replication load in Scribe have been identified as the rendezvous points (RP). An appropriate treatment of such service nodes becomes more important under the aspect of unbalanced packet replication, but poses a severe conceptual problem in DHTs: The placement of this entity should account for node and network capacities, but in a DHT is bound to the structural mapping of the multicast group identifier to an overlay key. Any alternative approach, e.g., selecting the RP address independently of the group address, will break the key space semantic with the result that an overlay node cannot derive the RP distribution address automatically.

Our prefix-guided broadcast strictly adheres to forwarddirected establishment of distribution trees. We have shown the generation of efficient group communication structures. The presented approach is thus particularly promising for overlay multicast services. Having sketched a structured multicast solution operating in prefix-space [15], its elaboration is subject to our currently ongoing work. Further on, we will integrate our scheme in hybrid group communication architectures [17].

\section{References}

[1] I. Baumgart, B. Heep, and S. Krause. OverSim: A Flexible Overlay Network Simulation Framework. In M. Faloutsos et al., editors, Proc. of the 10th IEEE Global Internet Symposium, pages 79-84, Washington, DC, USA, 2007. IEEE.

[2] I. Baumgart, B. Heep, S. Krause, and S. Mies. The OverSim P2P Simulator. http://www.oversim.org, 2008.

[3] M. Castro, P. Druschel, A.-M. Kermarrec, and A. Rowstron. SCRIBE: A large-scale and decentralized application-level multicast infrastructure. IEEE JSAC, 20(8):100-110, 2002.

[4] M. Castro, M. Jones, A.-M. Kermarrec, A. Rowstron, M. Theimer, H. Wang, and A. Wolman. An Evaluation of Scalable Application-level Multicast Built Using Peer-topeer Overlays. In IEEE Infocom 2003, volume 2, pages 1510-1520, Piscataway, NJ, USA, March 2003. IEEE Press.

[5] S. El-Ansary, L. A. Alima, P. Brand, and S. Haridi. Efficient Broadcast in Structured P2P Networks. In F. Kaashoek and
I. Stoica, editors, Proc. of IPTPS 2003, vol. 2735 of LNCS, pages 304-314. Springer-Verlag, Berlin Heidelberg, 2003.

[6] A. Ghodsi, L. O. Alima, S. El-Ansary, A. Ghodsi, P. Brand, and S. Haridi. Self-Correcting Broadcast in Distributed Hash Tables. In Proc. of 15th PDCS, 2003. ACTA Press.

[7] J. Li, K. Sollins, and D.-Y. Lim. Implementing Aggregation and Broadcast over Distributed Hash Tables. SIGCOMM Comput. Commun. Rev., 35(1):81-92, 2005.

[8] W. Li, S. Chen, P. Zhou, X. Li, and Y. Li. An Efficient Broadcast Algorithm in Distributed Hash Table Under Churn. In Proc. of WiCom'07, pages 1929-1932, Washington, DC, USA, 2007. IEEE Computer Society.

[9] P. Merz and K. Gorunova. Efficient broadcast in P2P Grids. In Proc. of the Fifth IEEE CCGrid, volume 1, pages 237242, Washington, DC, USA, 2005. IEEE Computer Society.

[10] S. Ratnasamy, M. Handley, R. M. Karp, and S. Shenker. Application-Level Multicast Using Content-Addressable Networks. In J. Crowcroft and M. Hofmann, editors, Proc. of the 3rd International COST264 Workshop, NGC'01, vol. 2233 of LNCS, pg. 14-29, London, 2001. Springer-Verlag.

[11] A. Rowstron and P. Druschel. Pastry: Scalable, distributed object location and routing for large-scale peer-to-peer systems. In IFIP/ACM Middleware, pages 329-350, Nov. 2001.

[12] I. Stoica, R. Morris, D. Karger, M. F. Kaashoek, and H. Balakrishnan. Chord: A scalable peer-to-peer lookup service for internet applications. In Proc. of SIGCOMM '01, pages 149-160, New York, NY, USA, 2001. ACM Press.

[13] A. Varga et al. The OMNeT++ discrete event simulation system. http://www.omnetpp.org, 2005.

[14] M. Wählisch. Scalable Adaptive Group Communication on Bi-directional Shared Prefix Trees. Technical Report TR-B08-14, Freie Universität Berlin, Department of Mathematics and Computer Science, Berlin, September 2008.

[15] M. Wählisch and T. C. Schmidt. Between Underlay and Overlay: On Deployable, Efficient, Mobilityagnostic Group Communication Services. Internet Research, 17(5):519-534, November 2007.

[16] M. Wählisch and T. C. Schmidt. Multicast Routing in Structured Overlays and Hybrid Networks. In X. S. Shen, H. Yu, J. Buford, and M. Akon, editors, Handbook of Peer-to-Peer Networking. Springer-Verlag, Berlin Heidelberg, 2009. In press.

[17] M. Wählisch, T. C. Schmidt, and G. Wittenburg. A Generalized Group Communication Network Stack and its Application to Hybrid Multicast. In Proceedings of the 28th IEEE INFOCOM. Student Workshop. IEEE Press, April 2009. 